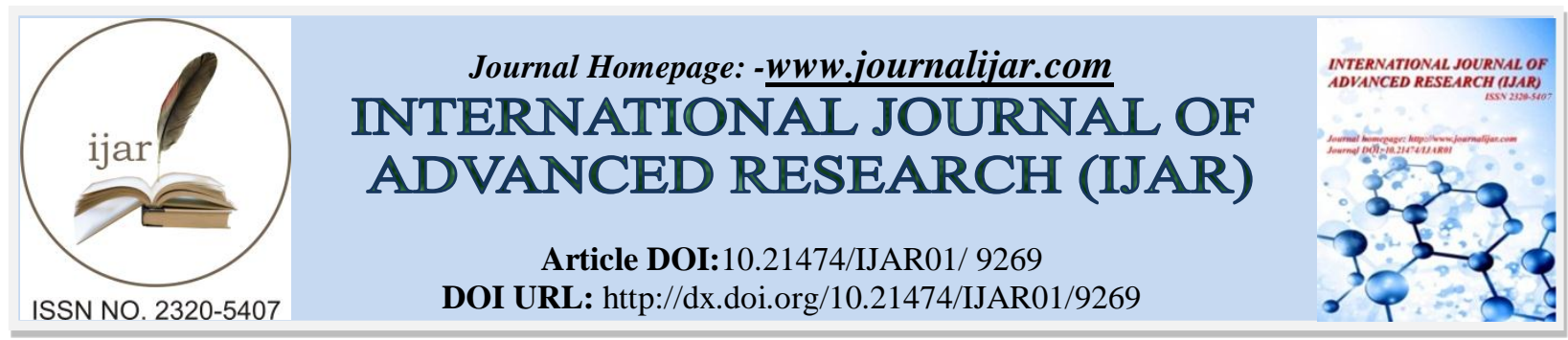

RESEARCH ARTICLE

\title{
SURGICAL ACCESS AND MANAGEMENT OF A RARE CASE OF GIANT COMPLEX COMPOSITE ODONTOMA IN THE MAXILLARY SINUS: A CASE REPORT.
}

\author{
Dr. Davide Rocchetta, Dr. Syed Sirajul Hassan, Dr. Faleh Ali AlShahrani, Dr. Bilal Al Masri, Dr. \\ Mohammed AlKindi.
}

\section{Manuscript Info}

Manuscript History

Received: 12 April 2019

Final Accepted: 14 May 2019

Published: June 2019

\section{Abstract}

\section{Introduction:-}

The term odontoma was first used by Broca in 1866, who defined it as a tumor constituted by tooth-forming tissues laid down with variable degrees of organization and mineralization. ${ }^{1}$ Actually odontomas are considered as hamartomas of aborted tooth formation rather than true neoplasms. ${ }^{2}$ They are composed of enamel, dentin, cementum and occasionally pulp tissue. ${ }^{3}$ Their pathogenesis is unclear and has been proposed to be associated with trauma during primary dentition, hereditary anomalies (Gardner's syndrome and basal cell nevus syndrome), odontoblastic hyperactivity or alterations of the genetics components responsible for controlling dental development. ${ }^{4}$ According to the latest World Health Organization (WHO, 2005) classification of odontogenic tumors, there are two main types of odontoma, composite and complex. ${ }^{5}$ Odontomas have also been classified as central or intraosseousodontoma (occur inside the bone), peripheral odontoma and erupted odontoma, that is quite rare. ${ }^{6}$ This article reports a rare presentation of a large erupted complex odontoma involving the right maxillary sinus treated surgically. It reviews also surgical access correlated with the removal of odontoma from the maxilla.

\section{Case report}

In October 2016, a 21-year-old female was referred to Oral and Maxillofacial Surgery, King Fahad Medical City, Riyadh, Saudi Arabia, for swelling in the right maxillary vestibule extending to the right eye. The patient was complaining of halitosis and pain irradiated in right cheek. At the intraoral examination the patient showed a firm swelling extending from the maxillary tuberosity up to the region of infra-orbital nerve, no deficit of sensitivity of the upper lip and cheek was present. The right upper second premolar and the first molar were mobile. The upper right second molar wasn't erupted and in its area there was a ulceration of oral mucosa with exposure of a greyish mass resembling bone or tooth exposed since long time in the oral cavity. At the extra oral examination a limited swelling in the right cheek was noticed and some submandibular nodes were enlarged probably due to the chronic inflammation. Panoramic radiographs revealed a solid radiopaque mass involving the right alveolar ridge, maxillary sinus, extending to the orbit (fig. 1). Axial and coronal reconstructed computed tomography images revealed a hyperdense mass extending from the alveolar ridge to the orbital floor involving the upper right second molar. The orbital floor was affected by the mass and in its anterior part there was a limited resorption (fig. 2A-2B). The differential diagnosis for the lesion includes complex odontoma, calcifying odontogenic cyst, calcifying odontogenic tumor, ameloblastic fibroma, fibro-odontoma, and fibro-osseous lesion. 7An incisional biopsy was performed with result of chronic osteomyelitis.

Patient was scheduled for excisional biopsy on general anesthesia. One hour prior to surgery patient was given 
dexamethasone $8 \mathrm{mg}$ intravenous (IV) and clindamycin 900mg IV, extraoral and intraoral antisepsis was performed with polyvinylpyrrolidone-iodine $2.0 \%$ and chlorhexidine $0.12 \%$ rinse for $1 \mathrm{~min}$, respectively. The operation was performed with the patient under hypotensive general anesthesia. The surgical procedure was initiated with an intrasulcular incision, from the maxillary right first incisor until the first molar and continued on the maxillary tuberosity. A relaxing incision was performed on the mesial of the incisor to allow themucoperiosteal flap to raise and expose the mass. In some parts the anterior surface of the maxillary bone was resorbed by the mass (fig. 3) and a complete surgical exposure was obtained with a round bur, the lesion was to big to excise in one block, so intraoperative decision was taken to divide the mass in multiple blocks till its complete removal (fig. 4). Once the mass was fully removed, a maxillary sinus exploration with endoscopic fiber optic to asset the orbital floor, that was intact and no defect was noticed. All the blocks removed were submitted for histopathological examination in formalin. After mass removal, the bony border of the surgical cavity were totally smoothened anda large communication between maxillary sinus and oral cavity was present, so harvested and traslated the buccal fat pad flap to close the deepest layer of the wound close to the maxillary tuberosity (fig. 5), the fat flap is sutured to the anterior wall of the maxillary sinus and to the palatal side to the mucosa (fig. 6), the mucosal flap was then repositioned and closed with interrupted stitches (fig. 7). Subject was prescribed analgesics paracetamol 1000mg every 8 hours for 7 days, clindamycin $600 \mathrm{mg}$ IV every 8 hours for 5 days. To the patient were taught sinonasal precautions for 2 weeks in order to avoid reopening of the oro antral fistula. The area healed uneventfully. Histopathological examination of the excised mass showed irregularly arranged dental hard tissues with areas of cell rich pulpal tissue. Clear spaces and clefts representing the mature enamel that is lost in the process of decalcification are often seen confirming the diagnosis of a complex odontoma. After 2 years after the lesion removal, panoramic radiographic demonstrated partial occurrence of bone repair in the treated area and did not reveal any signs of recurrence (fig. 8).

\section{Discussion:-}

Odontomas are considered as hamartomas of aborted tooth formation rather than true neoplasms, they are characterized by slow growth and nonaggressive behaviour ${ }^{6}$. The etiology of odontoma is not known but environmental causes such as infection, trauma, family history and genetic mutation are hypothesized. ${ }^{7}$ World Health Organization (WHO) has classified odontomas into three groups: complex odontoma, the calcified dental tissues are simply arranged in an irregular mass showing no morphological similarity to rudimentary teeth; compound odontoma, composed of all odontogenic tissuesresulting in many teeth-like structures, but without morphological resemblance to normal teeth; ameloblastic fibro-odontoma, consists of varying amounts of calcified dental tissue and dental papilla-like tissue, mostly resembling an ameloblastic fibroma. The ameloblastic fibro-odontome is considered as an immature precursor of complex odontoma. ${ }^{5}$ Odontomas are also classified as intraosseous and extraosseous. The intraosseousodontomas occur inside the bone and occasionally may erupt into the oral cavity (eruptedodontome). The extraosseous, also called peripheral odontomas, are odontomas occurring in the soft tissue covering the tooth-bearing portions of the jaws and having a tendency to exfolitate. ${ }^{8}{ }^{8}$ The odontoma constitute till $30 \%$ of all odontogenic tumors and the complex one is mostly found in the posterior mandible while the composite one in the anterior maxilla. ${ }^{1}$ In literature there have been isolated reports of odontomas affecting the maxillary sinus. ${ }^{7}$ A rare situation is when the odontoma erupts, i.e., when it becomes exposed through the soft tissues of the oral cavity. Rumel et al. in 1980 was the first who described the erupted odontoma. ${ }^{10}$ Since then only $30-40$ cases were reported in the literature. Theodontomaare seen with unerupted teeth in $10-44 \%$. Females are interested/affected little more than male (1.5:1). Majority of cases (84\%) are seen below 30 years but are seen in any age group with peak incidence in second decade. ${ }^{11,12,13}$

Most odontoma are asymptomatic and mostly are incidental findings on routine radiographs. Sometimes they can cause retention of deciduous teeth, unerupted permanent teeth, cortical expansion, neurosensory deficit and teeth mobility/displacement,they are all indicators for the presence of a possible odontoma ${ }^{12}$. Few cases are reported in literature with odontoma associated to swelling, infection or lymphadenopathy ${ }^{14,15}$. In our case the patient showed alitosis, pain irradiated to the right cheek and mobile molars close to the odontoma, even thought there wasn't neurosensory deficit. The radiographic characteristics of odontomas are mostly very useful to validate the diagnosis. The lesion consists of well-defined borders of a similar density to calcified dental tissue, this irregular radiodense mass is surrounded by a radiolucent halo, which usually represents an enlarged cystic follicle ${ }^{11}$. In composite odontoma are seen multiple teeth-like structures of varying size and shape. Complex odontomas are seen as irregular radiodense masses with no resemblance to dental structures. Histologically, odontomas comprise varying amount of enamel, pulp tissue, enamel organ and cementum. The connective tissue capsule is similar to that of dental follicle ${ }^{1}$. The management of the odontomas can be serial follow-up or surgical, with the last one more diffused. A removal 
and surgical curettage is mandatory to remove entirely the mass. The treatment can be different if we consider the compound type compared to the complex one. The first is usually easily removed under local anesthesia with a small mucoperiosteal flap like removing impacted teeth. The surgical treatment of the second one can be very challenging related mostly to the size and the position of the odontoma, in particular for the ones close to the maxillary sinus and especially regarding the surgical access. Traditionally the surgical approach to remove benign tumor from the maxillary sinus isthe trans-oral approach with Caldwell-Luc antrostomy through the antero-lateral wall of the maxillary sinus. It provides good surgical access with no cutaneous scar. By some authors has been advocated that this access could create a big defect in the anterior wall of the maxillary sinus with the risk of having soft tissue collapse and possible problems such as facial neurologic pain and numbness. ${ }^{16}$ These authors propose the maxillary lateral antrotomy with bone flap repositioning and fixation with a titanium plate to reduce the risk of having bony collapse restoring the anterolateral wall of the maxillary sinus immediately, but according to them this access can be used for medium size lesions, up to $3 \mathrm{~cm}^{16}$ and when the odontoma is not interesting the anterolateral wall itself with different possible degrees of bone resorption.

Korpi et al. in 2009 proposed the use of Le Fort I downfracture for removal of complex odontoma in the maxillary sinus and around its posterolateral wall. They suggest that this approach decreases the risk of bony defects, thus preventing oroantral fistula formation, and reduces facial deformity. ${ }^{17,18}$ The maxilla can be repositioned in its original position with titanium miniplates and screws. According to our experience this technique can be considered too aggressive for benign pathology and especially can create some problems and less reproducibility and stability in the result when large portion of maxillary walls are resorbed due to the growing of odontoma.

Murphy et al. in 2014 described the use of an upper lip split incision in order to facilitate complete removal of the tumour and maintain the integrity of the orbital floor and allow safe dissection and release of the infraorbital nerve. ${ }^{19}$ By some other authors, including us, it's not accettable to split upper lip to eradicate benign pathology and especially is not useful to visualize the orbital floor, which will be visualized with other types of facial access even more extensive (like for example Weber-Ferghusson access).

\section{Pitfalls and practical management}

Compound odontoma can be removed easily with simple mucoperiosteal flap like impacted teeth. For the complex ones, the surgical procedure can be more difficult and complicated. Usually is required general anesthesia or IV sedation because of the proximity of maxillary sinus or other anatomical structures, and because the great retraction needed on lips and cheeks to get enough exposure. The use of a bite block can be suggested if the lesion is in the maxillary sinus but if it's posterior isnot indicated because it pushes forward the coronoid process of the mandible and as a effect there is a limited mouth opening. Instead it can be used a large trapezoidal mucoperiosteal flap from anterior to the maxillary tuberosity to have great surgical view. Operatory lamps are difficult to use in such a small surgical space so its better the use of a fiberoptichead lamp. During the dissection of the mucosal flap is mandatory don't breach the periosteum to avoid the erniation of the buccal fad pad inside the surgical field with increase of difficulty and operating time.

Once that the exposure of the anterolateral wall of the maxillary sinus is completed an electric drill, bur shaped, can be used to create an opening starting from the points where the bone is thinner due to the growing of the odontoma, we need to remind that the window has to be smaller than the odontoma and once gained a correct exposure can be possible approach the odontoma in two different ways: try to remove it in one piece, reserved for the smaller ones but it can be very difficult and destructive for the surrounding tissues, or instead can be removed piece by piece considering that it's a benign pathology. Infact cutting the mass in small multiple pieces can have several advantages: less traumatic for the surrounding tissues, small bone window for the access to the maxillary sinus, less operative time because no need to expose all the mass before to start the removal procedure.

Another key-point is to save a certain amount of bone close to the maxillary tuberosity to increase the distal bone close to the most anterior tooth, this is possible if it's not interested by the mass like unfortunately in our case. In this way we'll be able to increase the prognosis of the last tooth with its roots surrounded by safe bone, at lest 1$2 \mathrm{~mm}$ will be enough to allow bone healing and regeneration. After the removal of the odontoma, considering that the access window is often smaller than the size of odontoma, it can be useful to inspect the maxillary sinus and the cavity with the endoscopy to check for any residual mass left.

At the end of the surgical procedure if the odontoma was involving the maxillary sinus, it's a frequent finding to 
have a oro-antral communication and it can be managed with the help of the buccal fat pad flap. If the Bichat flap isn't erniated yet during the dissection, a simple incision of the periousteum close to the first and second molar area, associated to divaricating of the opening with a mosquito in smooth way and gently push forward from the cheek can prepare the buccal fat pad for its use to close the oroantral communication in a second layer deeper to the mucoperiosteal one.

\section{Conclusion:-}

This case highlights a rare case of an erupting complex odontoma affecting the maxillary sinus and reviews the possible surgical access together to concerns and pitfalls for its management. The treatment of the odontoma is always surgical and considering the benign behavior special attention has to be considered when choosing the access for the surgery, it needs to be safe, with a reproducible result and asthethic.

Fig 1:-Preoperative panoramic showing Giant odontoma

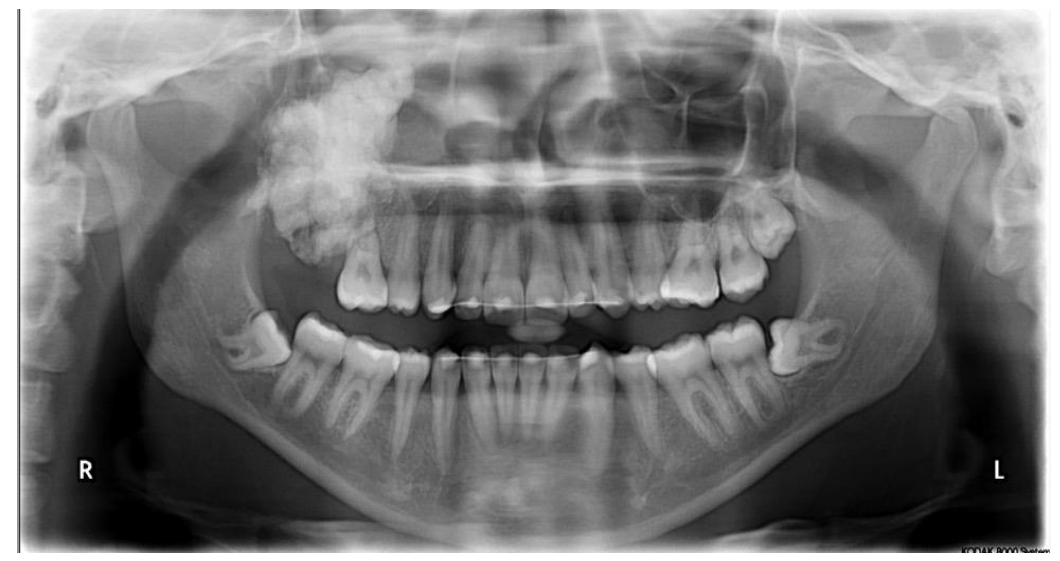

Fig. 2A:- CT scan: axial cut, in the right maxillary sinus there is a well defined mass which caused erosion of the posterolateral wall of the maxillary sinus itself

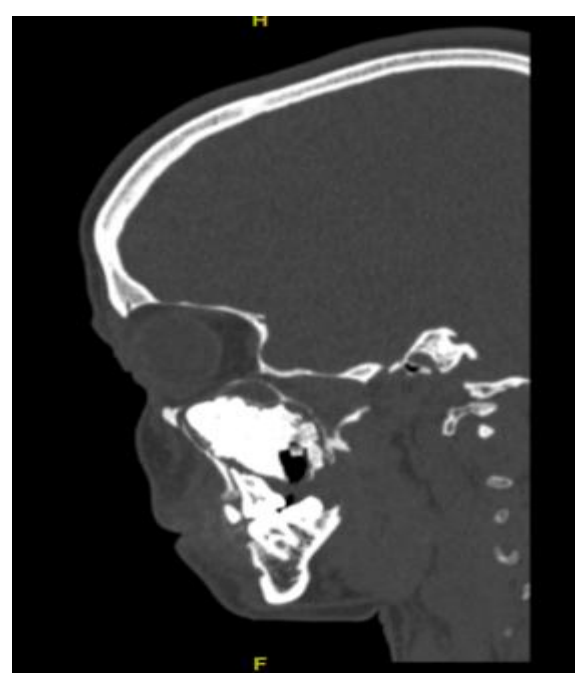




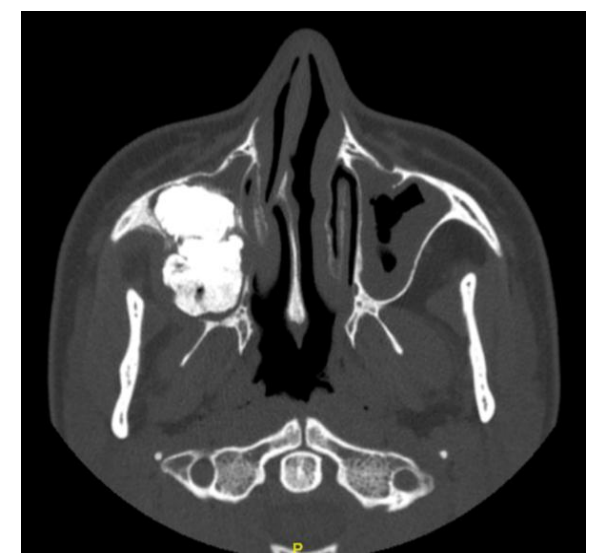

Fig 2B:-CT scan, sagittal cut: Entire right maxillary sinus is filled with the odontoma mass, extending from the tuber maxillae to the orbital floor

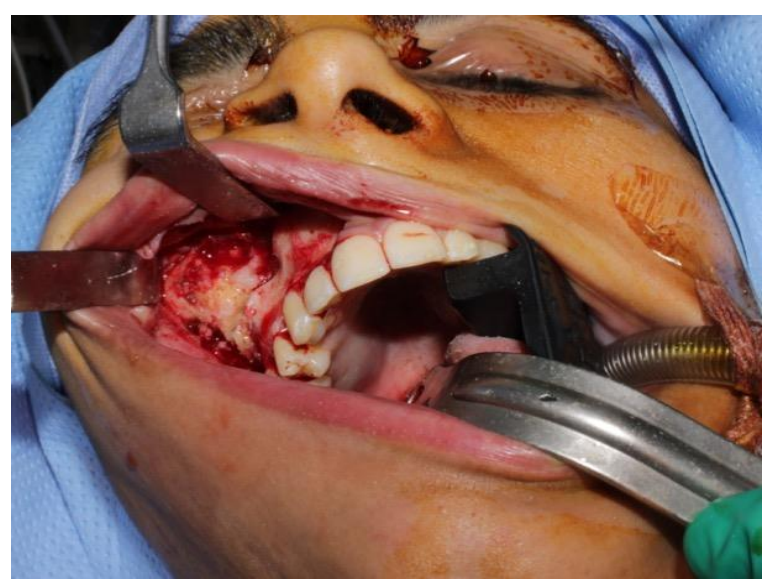

Fig 3:-Elevation of the mucoperiosteal flap appears that the odontoma has caused resorption of part of the lateral wall of the maxillary sinus

Fig 4:-Odontoma removed piecemeal: the biggest fragment is $2 \times 2 \mathrm{~cm}$

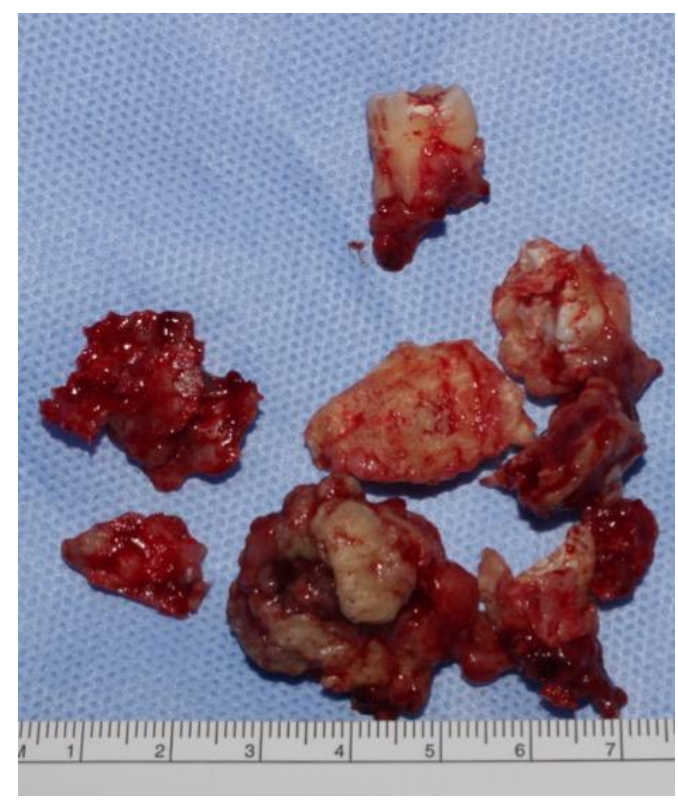




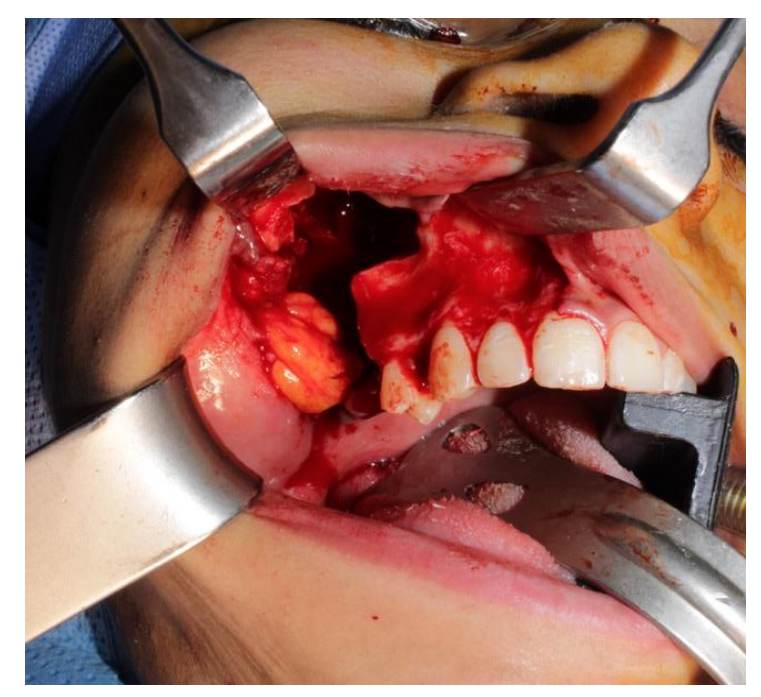

Fig 5:- Post removal of the odontoma, a large oroantral communication exist, the buccal fad pad flap is prepared to be rotated to close in a double layer the wound

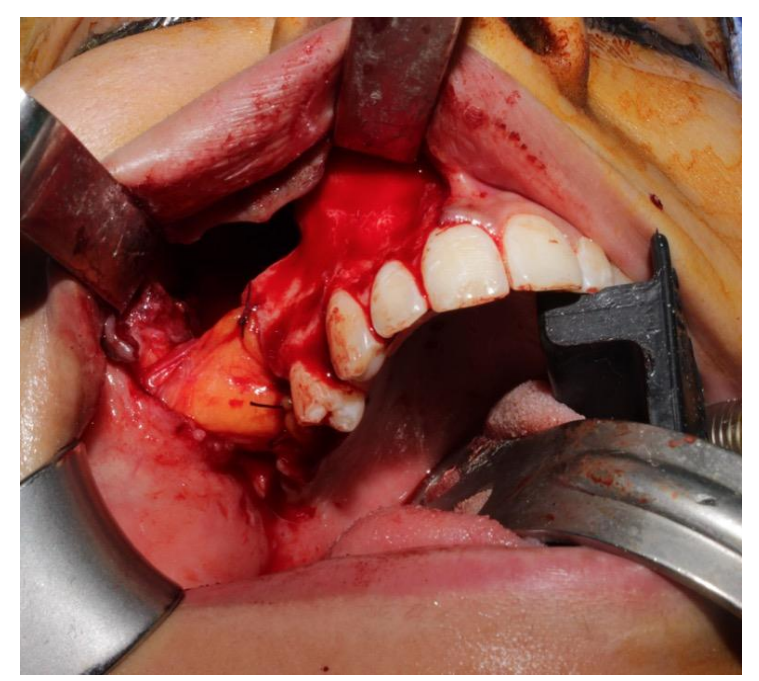

Fig 6:-The buccal fad pad flap has been sutured to the bone and to the soft tissue on the palatal side 


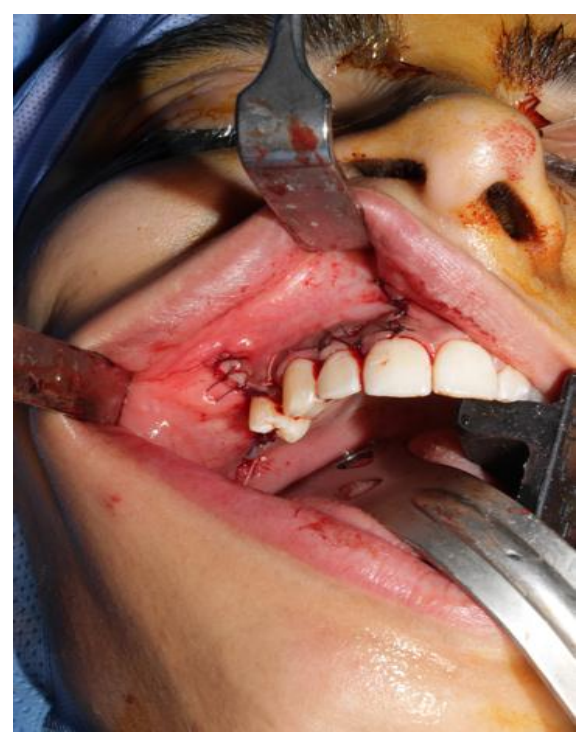

Fig 7:-Final view of the wound after double layer suturing has been done

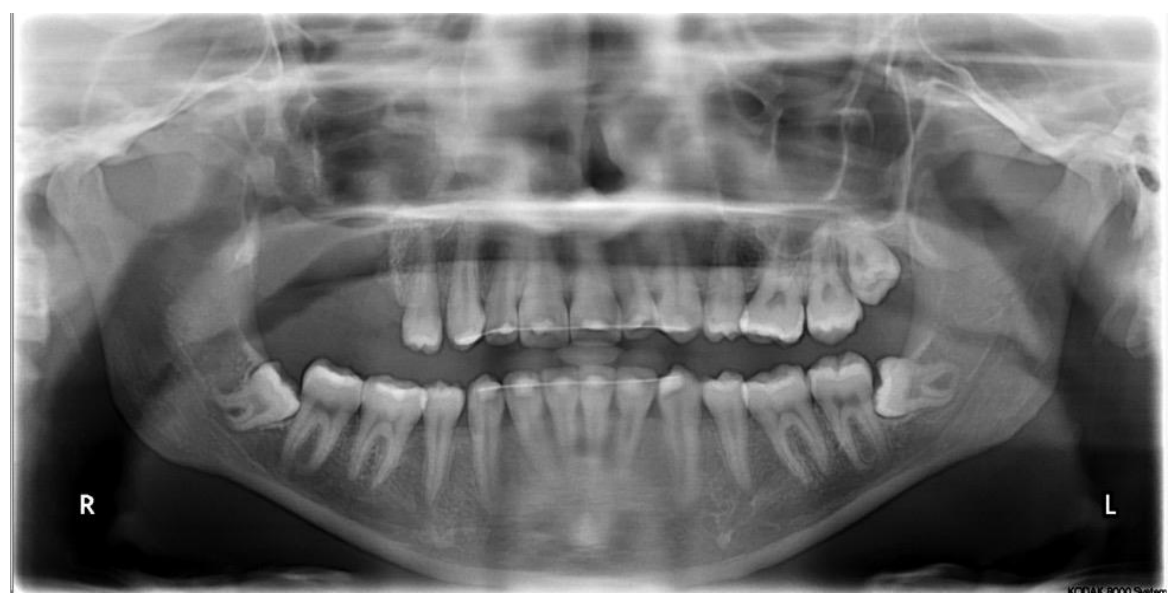

Fig 8:- Postoperative panoramic showing complete removal of odontoma

\section{References:-}

1. Cohen DM, Bhattacharyya I. Ameloblastic fibroma, ameloblastic fibro- odontoma, and odontoma. Oral Maxillofacial SurgClin N Am 2004;6:375- 384.

2. Shekar SE, Roopa SR, Gunasheela B, Supriya N. Erupted compound odontoma. J oral MaxillofacPathol 2009;13(1):47-50.

3. C. J. Perumal, A. Mohamed, A. Singh, and C. E. E. No e, "Sequestrating giant complex odontoma: a case report and review of the literature," Journal of Oral and Maxillofacial Surgery, vol. 12, no. 4, pp. 480-484, 2013.

4. Hitchin AD. The etiology of calcified composite odontoma. Br Dent J 1971;130:475-482.

5. Barnes L, Eveson JW, Reichart P, Sidransky D. Lyon: IARC; 2005. Pathology and genetics of the head and neck tumors. World Health Organization Classification of Tumors.

6. Junquera L, de Vicente JC, Roig P, Olay S, Rodriguez-Recio O. Intraosseous odontoma erupted into the oral cavity: An unusual pathology. Med Oral Patol Oral Cir Bucal 2005;10:248-251.

7. Mupparapu M, Singer SR, Rinaggio J. Complex odontoma of unusual size involving the maxillary sinus: report of a case and review of CT and histopathologic features. Quintessence Int 2004; 35(8):641-645.

8. Singh S, Singh M, Singh I, Khandelwal D. Compound composite odontoma associated with an unerupted deciduous incisor- A rarity. J Indian SocPedodPrev Dent. 2005;23:146-50.

9. Das UM, Nagarathna, ArathiUnerupted maxillary primary canine associated with compound composite odontoma. A case report. J Indian SocPedodPrev Dent. 2002;20:98-101.

10. Rumel A, de Fritas A, Birman E, Tannous L, Chacon P, Borkas S. Erupted complex odontoma. Report of a case. DentomaxillofacRadiol. 1980;9:5-9 
11. Vengal M, Arora H, Ghosh S, Pai KM. Large erupting complex odontoma: A case report. J Can Dent Assoc. 2007;73:169-73.

12. Reichart PA, Philipsen HP. Complex odontoma, odontogenic tumors and allied lesions. New Delhi: Quintessence Publishing Co; 2005. pp. 141-7.

13. Chang JY, Wang YP, Liu BY, Sun A, Chiang CP. Odontoma: A clinicopathologic study of 81 cases. J Formos Med Assoc. 2003;102:876-82.

14. Hidalgo-Sanchez O, Leco-Berrocal MI, Martinez-Gonzalez JM. Meta- analysis of the epidemiology and clinical manifestations of odontomas. Med Oral Patol Oral Cir Bucal. 2008 Nov 1;13(11):E730-734.

15. Al-Sahhar W, Putrus S. Erupted odontoma. Oral Surg Oral Med Oral Pathol. 1985;59:225-226.

16. Scolozzi P, Martinez A, Lombardi T, et al: Lateral antrotomy as a surgical approach for maxillary sinus: A modified technique with free bone flap repositioning and fixation with a titanium plate. J Oral MaxillofacSurg 67:689, 2009

17. J. T. Korpi, V. T. Kainulainen, G. K. B. Sándor, and K. S. Oikarinen, "Removal of large complex odontoma using Le fort 1 Osteotomy," Journal of Oral and Maxillofacial Surgery, vol. 67, no. 9, pp. 2018-2021, 2009.

18. Scolozzi P, Lombardi T. Removal of large complex odontoma using Le Fort I osteotomy. J Oral Maxillofac Surg. 2010;68:950-1.

19. Murphy C, O’Connel JE, Cotter E, Kearns G Management of Large Erupting Complex Odontoma in Maxilla. Case Rep Pediatrics, 2014, online published. 